\title{
Breast Curvature of the Upper and Lower Breast Mound: 3D Analysis of Patients who Underwent Breast Reconstruction
}

\author{
Juhun LEE ${ }^{a, b}$, Gregory P. REECE ${ }^{b}$, Mia K. MARKEY ${ }^{\mathrm{a}, \mathrm{b}^{*}}$ \\ ${ }^{a}$ The University of Texas at Austin, Austin (TX), USA; \\ ${ }^{\mathrm{b}}$ The University of Texas MD Anderson Cancer Center, Houston (TX), USA;
}

\begin{abstract}
Quantitative and objective methods to evaluate the morphology of the reconstructed breast may help plastic surgeons improve their surgical practice, and thus ultimately help breast cancer survivors derive the intended psychosocial benefits of reconstruction. Recently, we developed a quantitative and objective way to measure the curvature of the breast on standard clinical photographs. Here we conduct a more-in depth analysis of breast curvature using 3D images of the human torso. The analysis is based on the catenary curve, which is a perfectly flexible and inextensible string of uniform density supported by two distinct points. In this study, we used our curvature measure to study the upper and lower curvature of the breasts (breast curvature) of 9 patients who underwent breast reconstruction surgery. Breast reconstruction was performed using the autologous flap in 4 patients (5 breasts) and a tissue expander/implant (TE/Implant) in 5 patients (6 breasts). To assess the breast curvature, the outlines of the upper and lower breast were first obtained from coronal sectional views that are created from multiple parallel planes to the chest wall, which are spaced about $1 \mathrm{~cm}$ apart from each other. Then the curvature measure was extracted from the catenary contours fitted on the resulting outline of each sectional view. Our data show that breast curvatures of the upper and lower breast mound vary from the most anterior part of the breast mound to the chest wall. Moreover, our data suggest possible trends on the upper and lower breast curvature for different reconstruction types. For example, the opposite relationship was found between the upper and lower breast curvature for autologous and TE/Implant reconstructed breasts. In this study, we demonstrated a breast curvature measure for evaluating the outcome of breast reconstruction, especially for the curvature of the upper and lower breast mounds, based on 3D torso images of patients.
\end{abstract}

Keywords: Catenary, Breast curvature, Breast cancer, Breast reconstruction, 3D stereophotogrammetry

\section{Introduction}

Breast cancer is the one of the most common types of cancer for women residing in U.S. The estimated number of new cases of breast cancer in the U.S. in 2012 is 226,870 [1]. Fortunately, the death rate due to breast cancer has been decreasing since late 1980s thanks to improved early detection techniques and cancer treatments such as medical adjuvant and neo-adjuvant therapies. Since then, there is increasing emphasis on the quality of life of breast cancer survivors. In this respect, the goal of the breast reconstruction is to provide breast cancer survivors a breast form that is satisfying to them to aid in their psychosocially adjustment.

Until recently, the evaluation of breast reconstruction aesthetic outcomes was solely dependent on the surgeon's qualitative and subjective assessment of the shape of the reconstructed breast. Quantitative and objective methods to evaluate the morphology of the reconstructed breast may help plastic surgeons improve their surgical practice, and thus ultimately help breast cancer survivors derive the intended psychosocial benefits of reconstruction.

Three-dimensional (3D) imaging of the breast has drawn much attention in the field of plastic and reconstructive surgery in the last decade, due to its ability to allow users to evaluate the entire exposed skin surface area of the breast (except in patients with significant ptosis) in real units (e.g., in $\mathrm{mm}$ ).

Many previous studies [2-7] utilized 3D imaging technique for assessing the breast morphology. However, the analyses of those previous studies were limited to: 1) simple breast volume difference, or 2) asymmetry by superimposing one breast image over another. To the best of our knowledge, no study has analyzed the curvature of the breast mound using the 3D torso images of women. Kawale et al. [7] evaluated the influence of subject's pose (arms-rest pose vs. hands-on-hip pose) during 3D image acquisition on the asymmetry of the breast morphology. Losken et al. [2] assessed the asymmetry of the breast of women without history of breast cancer using 3D images of their torso.

*mia.markey@utexas.edu; +1-512-471-1711; bmil.bme.utexas.edu 
They divided the subject's torso image into left and right halves using the midline of the subject's torso and assessed the asymmetry by computing the root mean squared error between them by mirroring the left half to the right half. In the study of Galdino et al. [3], the authors reported 5 case studies in which they evaluated the morphology of the breast using 3D imaging after various types of breast surgery including breast reconstruction. They reported the change of the breast shape using quantitative measures such as the vertical surface distance between the nipple and the inframammary fold and the volume of the breast. In addition, Isogai et al. [5] quantitatively compared the shape of post-reconstructed breast of patients who underwent unilateral breast reconstruction to that of the natural breast of the same patient using Moire patterns on the patient's 3D torso image. Moreover, Nahabedian and Galdino [4] utilized 3D imaging technique to compute the volume of the reconstructed breasts. In the study of Tepper et al. [6], the asymmetry of the breasts of patients who underwent unilateral Tissue Expander (TE)/Implant reconstruction was evaluated using the 3D torso image of the patients.

Recently, we developed a quantitative and objective way to measure the curvature of the breast on standard clinical photographs [8]. The measure can be easily extended to 3D surface images of patients. In this study, we extended our curvature measure to evaluate the upper and lower curvature of the breasts (breast curvature) of 9 patients who underwent breast reconstruction surgery at The University of Texas MD Anderson Cancer Center.

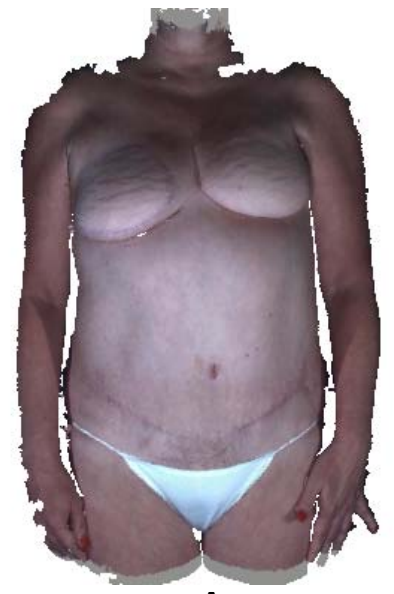

A

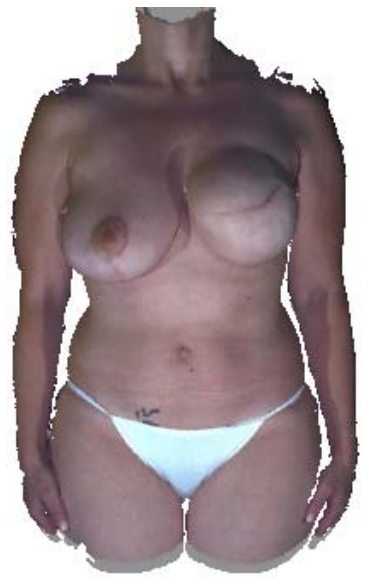

B

Fig. 1. Examples of $3 D$ surface images used for this study. Each $3 D$ image taken from patients standing comfortably erect with arms at sides. A: Bilateral DIEP reconstructed breast. B: Unilateral TE/Implant reconstructed breast (after placement of a permanent silicone-filled breast implant).

\section{Materials and Methods}

\subsection{Dataset}

The study population for this paper consists of women aged 21 or older who underwent or were scheduled for breast reconstruction surgery from April 1, 2011 to June 31, 2011 at The University of Texas MD Anderson Cancer Center. A 3dMDTorso system (3dMD, Atlanta, GA) was used to obtain 3D images of torsos of 9 patients. Breast reconstruction was performed using the autologous flap in 4 patients (total 5 reconstructed breasts) and a tissue expander/implant (TE/Implant) in 5 patients (total 6 reconstructed breasts). Figure 1 shows examples of the 3D images used for this study. The summary information of study patients is given in Table 1. Of 5 autologous flap reconstructed breasts, 2 were reconstructed with a Transverse Rectus Abdominis Muscle (TRAM) flap and 3 with a Deep Inferior Epigastric Perforators (DIEP) flap. Of $6 \mathrm{TE} / \mathrm{Implant}$ reconstructed breasts, 3 had undergone placement of a permanent implant and 3 were in the TE phase of the reconstruction. Among 3 reconstructed breasts in the implant phase, 2 were smooth round saline-filled implants and the other was smooth round silicone-filled implant. The saline-filled implants were undergoing over inflation of the implant by $15 \%$ of the manufacturers volume of the implant. Among the 3 breasts in the TE phase of reconstruction, 1 was over inflated by $33 \%, 1$ by $75 \%$, and 1 by $8 \%$ of the manufacturers volume of the TE. All 3D images are rotated 15 degree in the $x$-axis to compensate for the angle introduced during the image acquisition stage, which is due to the configuration of cameras of $3 \mathrm{dMDT}$ Torso system. 
Table 1. Summary information of study subjects

\begin{tabular}{|c|c|c|c|c|c|}
\hline \multirow{2}{*}{$\begin{array}{l}\text { Patient } \\
\text { Study ID }\end{array}$} & \multirow{2}{*}{$\begin{array}{l}\text { Breast } \\
\text { Side }\end{array}$} & \multirow{2}{*}{$\begin{array}{l}\text { Reconstruction } \\
\text { Type }\end{array}$} & \multicolumn{2}{|c|}{ TE/Implant Type } & \multirow{2}{*}{$\begin{array}{l}\text { Days from the last } \\
\text { reconstructive surgery }\end{array}$} \\
\hline & & & Product ID & $\begin{array}{c}\text { Filled } \\
\text { Volume }\end{array}$ & \\
\hline \multirow{2}{*}{150} & L & DIEP & NA & NA & \multirow{2}{*}{336} \\
\hline & $\mathrm{R}$ & DIEP & NA & NA & \\
\hline 167 & L & DIEP & NA & NA & 168 \\
\hline 157 & $\mathrm{R}$ & TRAM & NA & NA & 116 \\
\hline 169 & L & TRAM & NA & NA & 131 \\
\hline 178 & L & TE & $\begin{array}{c}\text { Allergen Textured } \\
\text { Style } 133 \mathrm{MV}-11\end{array}$ & $108 \%$ & 131 \\
\hline 179 & L & TE & $\begin{array}{l}\text { Allergen Textured } \\
\text { Style } 133 \mathrm{MV}-15\end{array}$ & $175 \%$ & 182 \\
\hline 180 & $\mathrm{R}$ & TE & $\begin{array}{l}\text { Allergen Textured } \\
\text { Style } 133 \mathrm{MV}-12\end{array}$ & $133 \%$ & 282 \\
\hline 152 & L & Implant & $\begin{array}{c}\text { Allergen Silicone } \\
\text { Smooth Round Style } \\
45-800\end{array}$ & NA & 160 \\
\hline \multirow{2}{*}{174} & L & Implant & $\begin{array}{l}\text { Mentor Saline Smooth } \\
\text { Round Spectrum } \\
350-1490\end{array}$ & $115 \%$ & \multirow{2}{*}{222} \\
\hline & $\mathrm{R}$ & Implant & $\begin{array}{l}\text { Mentor Saline Smooth } \\
\text { Round Spectrum } \\
350-1490\end{array}$ & $115 \%$ & \\
\hline
\end{tabular}

\subsection{Obtaining concentric upper and lower breast curvature}

In this study, we conducted a preliminary analysis of the curvature of the upper and lower breast. The analysis is based on the rotated catenary curve [8], which can be expressed as follows

$$
\left[\begin{array}{l}
x_{s} \\
y_{s}
\end{array}\right]=\left[\begin{array}{cc}
\cos \theta & -\sin \theta \\
\sin \theta & \cos \theta
\end{array}\right]\left[\begin{array}{c}
\alpha \cdot s+b \\
-\alpha \cosh (s)+c
\end{array}\right],
$$

where $b$ and $c$ are the offset of the $x$-axis and y-axis, respectively, and $s$ is a free parameter for the catenary curve, where its range governs the evolvement of the curve. Moreover, $\alpha$ is the ratio of the tension to the weight applied to each point on the curve. The shape of catenary is determined by $\alpha$, which is the curvature measure. A rotation parameter $\theta$ is introduced to capture the natural angle of the breast contour to the imaginary horizontal line. This angle is formed due to the shape of a person's chest wall and the weight of the breast. Figure 2 shows how $\alpha, s$, and $\theta$ govern the shape of the rotated catenary curve. As equation (1) and Figure 2 indicate, there is an inverse relationship between the value of $\alpha$ and the curvature of the curve; the curvature value of the curve decreases as $\alpha$ increases and vice versa.

To assess the breast curvature of the upper and lower breast mound, the outlines of the upper and lower breast were first obtained from coronal sectional views that are created from multiple parallel planes to the patient's chest wall, which are spaced about $1 \mathrm{~cm}$ apart from each other where it starts from the most anterior part of the reconstructed breast (i.e., the farthest point of the reconstructed breast from the coronal plane). We used the most anterior part of the breast mound as a starting point for the parallel planes since the nipple is typically missing in the process of the reconstruction unless patients underwent nipple-sparing mastectomy. Figure 3.A depicts concentric breast contours of a breast mound that are obtained from each sectional view. 


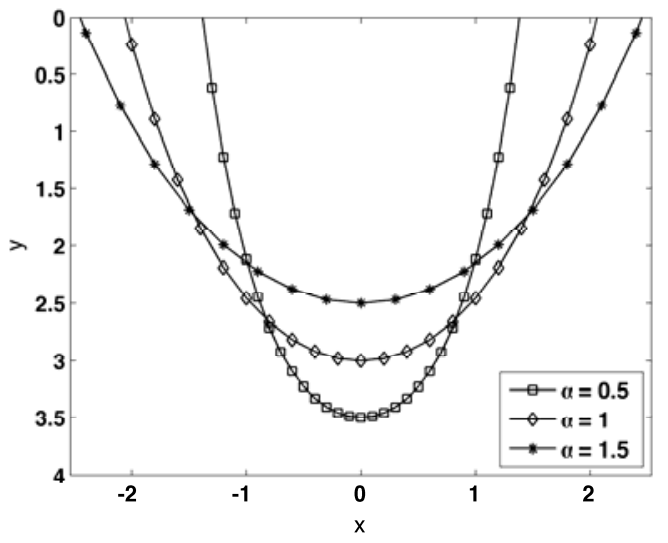

A

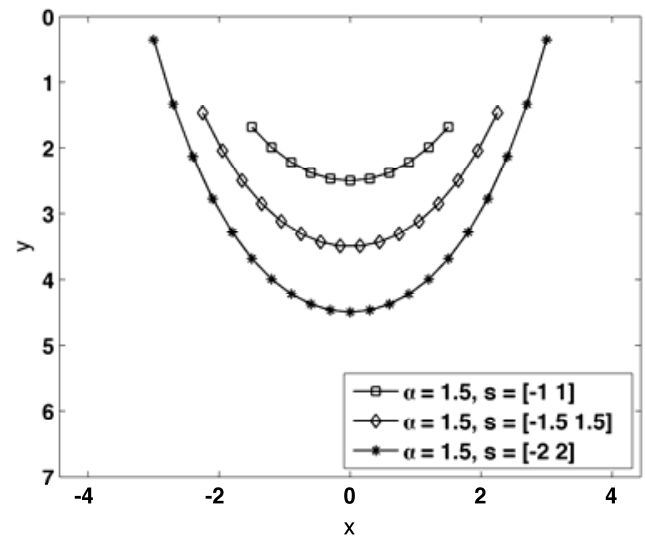

B

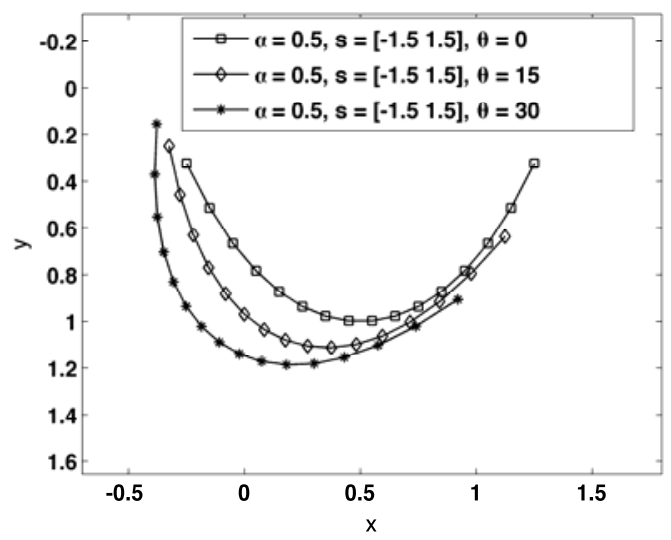

C

Fig. 2. This figure shows graphs of catenaries for different $\alpha$ values $(A)$, different the range of $s(B)$, and different $\theta$ values (C). A: a smaller $\alpha$ corresponds to a curve with higher curvature where as a larger $\alpha$ corresponds to a curve with lower curvature. B: the catenary evolves as the range of s increases. $\boldsymbol{C}$ : $\theta$ captures the orientation of the breast contour from the $x$-axis.

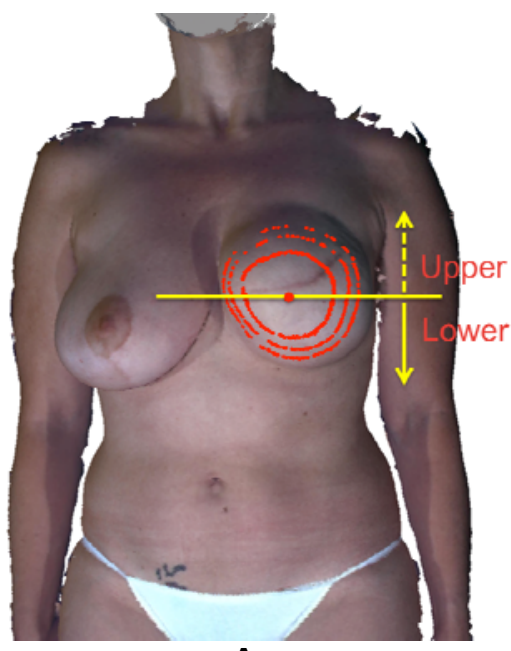

A

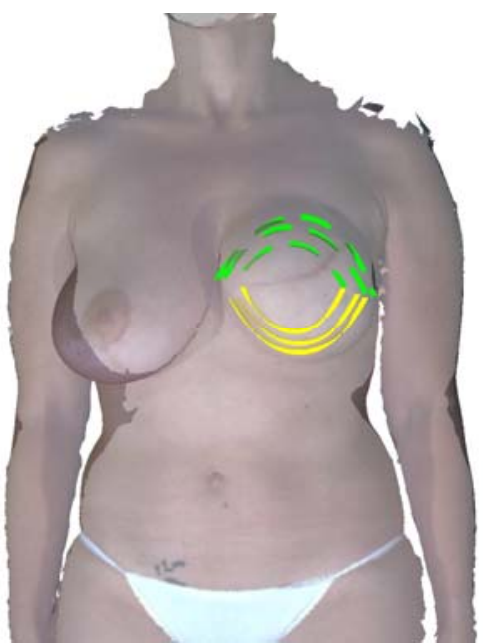

B

Fig. 3. A: Concentric breast contours of a breast mound. Starting from the top of the breast (red dot at the most anterior part of the breast mound), each concentric contour is obtained from coronal sectional views that are spaced about $1 \mathrm{~cm}$ apart from each other. The mathematical centroid of the breast outline on each sectional view was first obtained. In this image, the location of the mathematical centroid is coincided with the location of the most anterior part of the breast mound. Using the location of the obtained centroid, the breast outline is divided horizontally, which makes upper and lower concentric contours. The curvature measure was computed from these concentric breast contours. B: Concentric catenary curves fitted on the breast mound. The curvature measure $\alpha$ was extracted from the upper and lower catenary contour for each coronal sectional view. 
Once the sectional views of the breast mound are obtained, obtaining the curvature of the upper and lower breast mound is straightforward. We first obtain the mathematical centroid of the breast outline on each sectional view. Using the location of the obtained centroid, the breast outline is divided horizontally, which results in upper and lower concentric contours (Figure 3.A). Then we extract the curvature measure $\alpha$ from the catenary contours fitted on the resulting upper and lower part of the breast outline. This is equivalent to solving the following equation,

$$
\left[\begin{array}{l}
x \\
y
\end{array}\right]=F(s, P), P=[\alpha, \theta, b, c],
$$

where $F$ denotes the catenary curve on each part of the ellipse, i.e., (1), and $x$ and $y$ represent the $x$ and $y$-coordinates of the breast contour on the sectional view. Since the length of the breast outline on each sectional view is different from each other and varies across women, the different sets of ranges of $s$ should be used. In our previous study [8], we found that the catenary curve with $s=[-11]$ and $s=$ [-2.5 2.5] can be fitted to the breast contour of extremely small and large breast, respectively. Therefore, we selected [-1 1] and [-2.5 2.5] as the lower and upper bounds of the range of $s$, respectively. The bisection algorithm was used to find the appropriate range of $s$, which minimizes the error between the breast outline on the sectional view and the fitted catenary curve. In summary, we solved the following problem in the least square sense for both the upper and lower part of the ellipse on each sectional view,

$$
\min _{P_{j}} \sum_{j}\left(F\left(s_{i, j}, P_{j}\right)-\left[\begin{array}{c}
x_{i} \\
y_{i}
\end{array}\right]\right)^{2},
$$

where $x_{i}$ and $y_{i}$ are the coordinates of the ith point on the breast contour on the sectional view, and $s_{i, j}$ is the catenary point in $j$ th sets of the range of $s$, corresponding to the location of $\left[x_{i}, y_{i}\right]^{\top}$. The $P_{j}$ that minimizes (3) was set as a solution $P$. As a result, the catenary contours were arranged in a concentric fashion over the breast as shown in Figure 3.B.

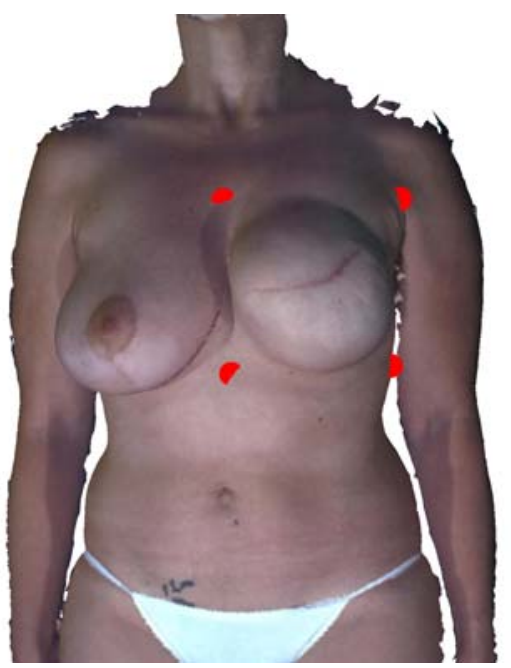

Fig 4. This figure illustrates 4 landmarks for computing the volume of the reconstructed breast. Two inferior points are on the midline of the subject's torso and the other twos are on the subject's anterior axillary line. The vertical locations of the landmarks were adjusted such that the Coons Patch included the breast mound.

Once the solution $P$ is found, we can obtain the breast curvature measure $\alpha$ for each part of the ellipse in each sectional view. To compare the curvature measure values of different individuals, we normalized the curvature measure $\alpha$ by the length of the fitted curve. We denote the normalized version of the curvature measure as $\alpha$. All analyses related to the curvature of the breast mound were performed using the MATLAB v.7.12.0 (R2011a) (The Mathworks, Natick, MA).

In addition to the analysis on the upper and lower curvature of the reconstructed breast mounds, we computed the volume of the reconstructed breasts using software (3dMDpatient, 3dMD, Atlanta, GA) that is provided with the imaging system. A total of 4 points surrounding the breast were manually located to create a Coons Patch, which approximates the surface of the subject's chest wall. We used the anterior axillary line and the midline on the subject's torso to locate those 4 points. The locations of those points were vertically adjusted such that the Coons Patch included the breast mound (Figure 4). 
Then, the program automatically computes the volume of the breast mound using the breast surface and the identified Coons Patch.

\section{Results}

Table 2 and Figure 5 summarize the upper and lower curvature values, and the computed breast volume for each study patient. The lower curvature value of patient 180 obtained from the sectional view at $3 \mathrm{~cm}$ was excluded from the study since the sectional view included part of the chest wall behind the breast mound.

Our data show that breast curvatures of the upper and lower breast mound vary from the most anterior part of the breast mound to the chest wall. For autologous reconstructed breasts (DIEP and TRAM flaps), our data suggest that the lower breast mound is slightly more curved than the upper breast mound when the patient is in a standing position. For TE/Implant reconstructed breasts, an inverse relationship between the lower and upper breast mound was observed when the patient was in the standing position. Moreover, our measure suggests a possible difference in curvature between large and small autologous (abdominal based) reconstructed breasts. That is, the reconstructed breasts with large volume (in this case, TRAM reconstructed breasts) are more curved than the reconstructed breasts with relatively small volume (in this case, DIEP reconstructed breasts).

Table 2. The upper and lower breast curvature values for the breast mounds of all study patients.

\begin{tabular}{|c|c|c|c|c|c|c|}
\hline \multirow{2}{*}{$\begin{array}{l}\text { Patient } \\
\text { Study ID }\end{array}$} & \multirow{2}{*}{$\begin{array}{l}\text { Reconstruction } \\
\text { Type / Computed } \\
\text { Volume }\end{array}$} & \multirow{2}{*}{$\begin{array}{l}\text { Breast } \\
\text { Side }\end{array}$} & & \multicolumn{3}{|c|}{$\begin{array}{l}\text { Breast curvature value of concentric } \\
\text { breast outline at each sectional view }\end{array}$} \\
\hline & & & & $1 \mathrm{~cm}$ & $2 \mathrm{~cm}$ & $3 \mathrm{~cm}$ \\
\hline \multirow[t]{4}{*}{150} & DIEP & L & Upper & 0.289 & 0.299 & 0.294 \\
\hline & Volume: $519 \mathrm{cc}$ & & Lower & 0.310 & 0.300 & 0.268 \\
\hline & DIEP & $\mathrm{R}$ & Upper & 0.300 & 0.301 & 0.294 \\
\hline & Volume: 548 cc & & Lower & 0.281 & 0.303 & 0.253 \\
\hline \multirow[t]{2}{*}{167} & DIEP & L & Upper & 0.279 & 0.266 & 0.266 \\
\hline & Volume: $592 \mathrm{cc}$ & & Lower & 0.296 & 0.265 & 0.304 \\
\hline \multirow[t]{2}{*}{157} & TRAM & $\mathrm{R}$ & Upper & 0.273 & 0.275 & 0.258 \\
\hline & Volume: $743 \mathrm{cc}$ & & Lower & 0.262 & 0.257 & 0.271 \\
\hline \multirow[t]{2}{*}{169} & TRAM & L & Upper & 0.252 & 0.276 & 0.287 \\
\hline & Volume: 886 cc & & Lower & 0.228 & 0.233 & 0.231 \\
\hline \multirow[t]{2}{*}{178} & TE & $\mathrm{L}$ & Upper & 0.230 & 0.215 & 0.155 \\
\hline & Volume: $415 \mathrm{cc}$ & & Lower & 0.208 & 0.239 & 0.219 \\
\hline \multirow[t]{2}{*}{179} & TE & L & Upper & 0.265 & 0.243 & 0.247 \\
\hline & Volume: 1127 cc & & Lower & 0.261 & 0.273 & 0.250 \\
\hline \multirow[t]{2}{*}{180} & TE & $\mathrm{R}$ & Upper & 0.248 & 0.244 & 0.265 \\
\hline & Volume: $271 \mathrm{cc}$ & & Lower & 0.257 & 0.301 & NA \\
\hline \multirow[t]{2}{*}{152} & Implant & L & Upper & 0.275 & 0.236 & 0.216 \\
\hline & Volume: 918 cc & & Lower & 0.242 & 0.270 & 0.263 \\
\hline \multirow[t]{4}{*}{174} & Implant & L & Upper & 0.281 & 0.286 & 0.280 \\
\hline & Volume: 894 cc & & Lower & 0.275 & 0.281 & 0.276 \\
\hline & Implant & $\mathrm{R}$ & Upper & 0.220 & 0.235 & 0.255 \\
\hline & Volume: 804 cc & & Lower & 0.260 & 0.270 & 0.254 \\
\hline
\end{tabular}



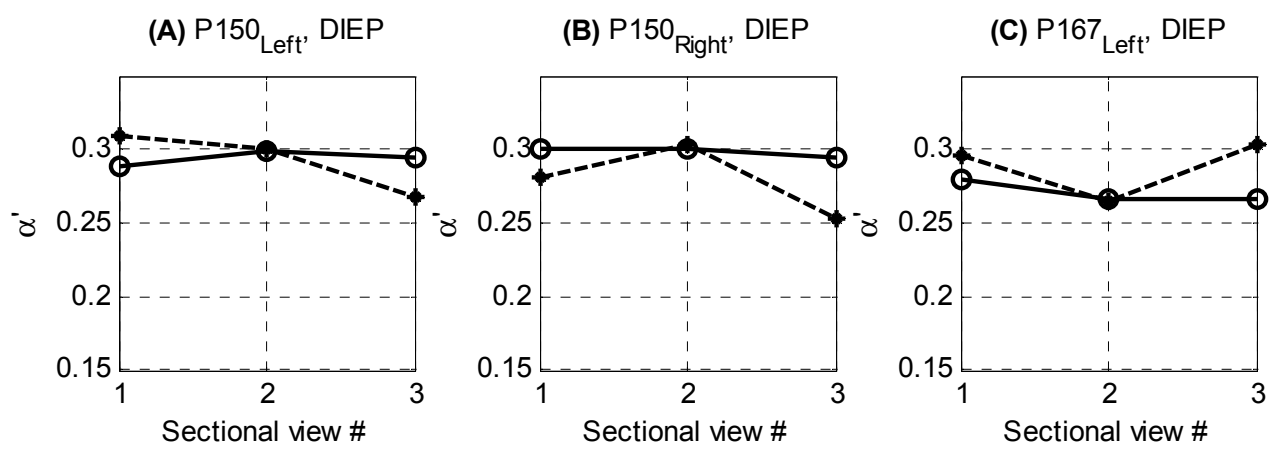

(D) P157 ${ }_{\text {Right' }}$ TRAM

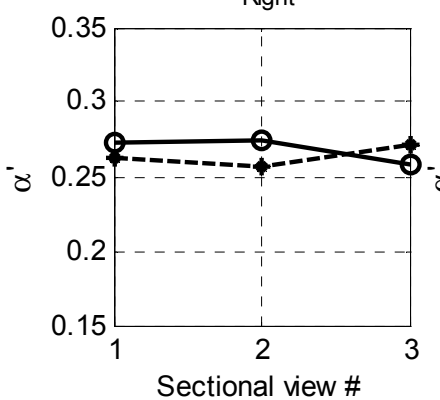

(E) P169 Left' TRAM
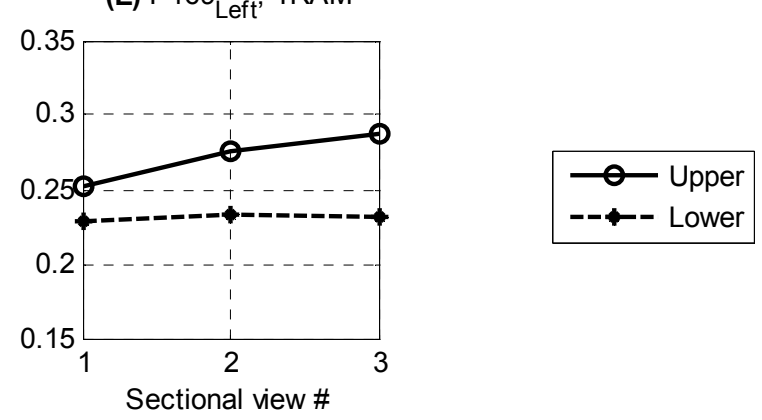

(F) P178 Left' TE

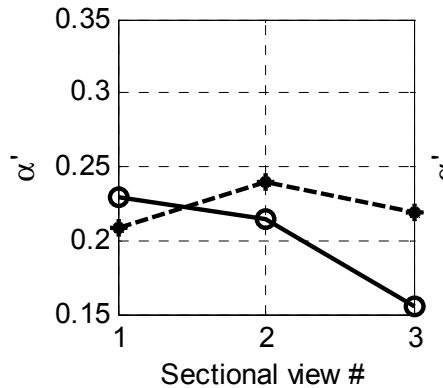

(G) P179 ${ }_{\text {Left }}$, TE

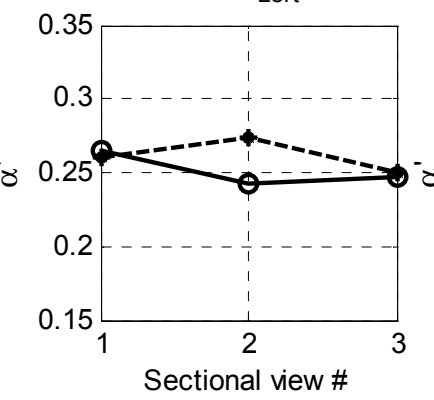

(H) P180 Right' TE
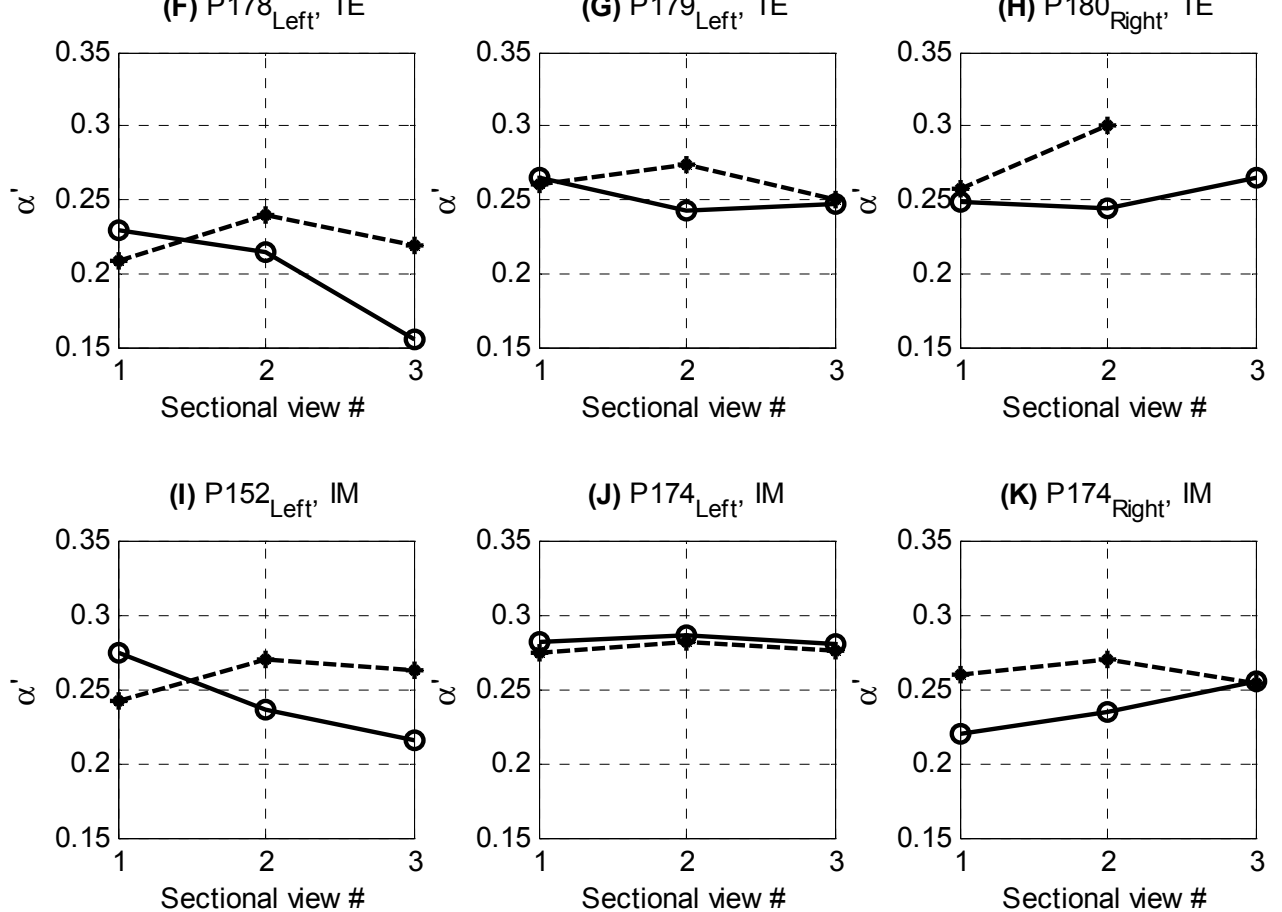

(K) P174 ${ }_{\text {Right }}$, IM

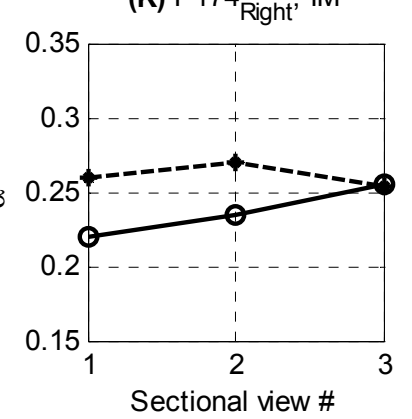

Fig. 5. Graphical illustration of the curvature values of upper and lower breast mounds of all study patients. Our measure suggests that for each sectional view, the lower half of the breast mound curvature of autologous reconstructed breasts (DIEP and TRAM flaps) is slightly more curved than the upper half of the breast mound curvature of the same breast, while an inverse relationship between the lower and upper breast mound curvatures was observed for TE/Implant reconstructed breasts. Moreover, our measure suggests a possible difference in curvature in that the reconstructed breasts with large volume (in this case, TRAM reconstructed breasts) are more curved than the reconstructed breasts with relatively small volume (in this case, DIEP reconstructed breasts). 


\section{Discussion}

In this study, we demonstrated the usage of our curvature measure for evaluating the upper and lower curvature of the breasts of patients who underwent breast reconstructive surgery. The curvature measure was obtained by fitting a catenary curve on concentric breast contours from coronal sectional views that are created from multiple parallel planes to the patient's chest wall, which are spaced about $1 \mathrm{~cm}$ apart from each other. Our data suggest possible trends in the upper and lower breast curvature for different reconstruction types. For example, the opposite relationship was found between the upper and lower breast curvature for autologous (abdominal based) and TE/Implant reconstructed breasts.

In addition, we showed that the curvature of the reconstructed breast mound changes from the most anterior part of the breast mound to the chest wall. There was no simple pattern (either monotonically increasing or decreasing) identified for the upper and lower breast contours of the reconstructed breast mound. This is reasonable since the shape of the breast mound is different from one woman to another.

However, although we cannot conclude that it is statistically significant, the sectional breast curvature values of the breast mound of the patient who had (Patient study ID \#174) bilateral reconstruction surgery with the same permanent implants are different from each other (Table 1). This curvature changes coincide with the difference on the computed volume (90cc difference, see Table 2). This is interesting since it is expected that the shape of implants with the same product ID (see Table 1) will be similar to each other. This difference may be due to the following reasons: 1) the shape difference of the left and right chest wall, 2) the thickness of the breast skin and subcutaneous tissue after mastectomy, 3) the existence of capsular contracture, and 4) the shape and location of the mastectomy scar.

The case example of the patient that underwent bilateral breast reconstruction with implants shows the difficulty of achieving symmetry and the necessity of using quantitative measures to assess breast morphology.

Future work building on this study will include conducting statistical analyses of the upper and lower breast curvature changes associated with: 1) different types of reconstruction techniques, 2) bilateral and unilateral reconstruction, and 3) natural and reconstructed breasts. From these future studies, we might be able to provide a more in depth understanding of the morphology of both natural and reconstructed breasts to surgeons.

In this study, we demonstrated a breast curvature measure for evaluating the outcome of breast reconstruction, especially for the curvature of the upper and lower curvatures of breast mounds, based on $3 \mathrm{D}$ torso images of patients.

\section{References}

1. (2012): "Cancer Facts and Figures 2012", American Cancer Society.

2. Losken, A., et al., (2005): "An Objective Evaluation of Breast Symmetry and Shape Differences Using 3-Dimensional Images.", Ann. Plast. Surg., 55(6): p. 571-575.

3. Galdino, G.M., et al., (2002): "Clinical applications of three-dimensional photography in breast surgery.", Plastic \& Reconstructive Surgery, 110(1): p. 58-70.

4. Nahabedian, M.Y. and G. Galdino, (2003): "Symmetrical breast reconstruction: is there a role for three-dimensional digital photography?", Plastic \& Reconstructive Surgery, 112(6): p. 1582-90.

5. Isogai, N., et al., (2006): "Quantitative analysis of the reconstructed breast using 3-dimensional laser light scanner.", Annals of Plastic Surgery, 56(3): p. 237-42.

6. Tepper, O.M., et al., (2008): "Three-dimensional imaging provides valuable clinical data to aid in unilateral tissue expander-implant breast reconstruction.", The Breast Journal, 14(6): p. 543-550.

7. Kawale, M., et al., (2011): "3D Symmetry Measure Invariant to Subject Pose During Image Acquisition." Breast Cancer: Basic and Clinical Research, 5: p. 131-142.

8. Lee, J., et al., (2012): "A Novel Quantitative Measure of Breast Curvature Based on Catenary.", Biomedical Engineering, IEEE Transactions on, 59(4): p. 1115-1124. 